\title{
Trombose de veia mesentérica superior: relato de caso
}

Superior mesenteric vein thrombosis: case report

Trombosis de vena mesentérica superior: reporte de caso

\author{
Talita Costa BARBOSA 1 \\ Amanda Oliva SPAZIANI ${ }^{1}$ \\ Stephanie Tiosso Fontes MONTEIRO² \\ Trícia Aline Ribeiro Pattini de SOUZA 3 \\ Gustavo Rivelli LAMBOGLIA 3 \\ Rodrigo João Lopes TAVEIROS 3 \\ Marcos Rogério MARQUES ${ }^{3}$ \\ Ana Carolina Borges GORGA ${ }^{4}$ \\ André Fraga RUEDA ${ }^{4}$ \\ Clerson Rodrigues MANAIA ${ }^{4}$ \\ Cleidjane Furtado REZENDE ${ }^{4}$ \\ Pedro Gomes BARBOSA JUNIOR ${ }^{4}$ \\ Leonardo FAIDIGA ${ }^{4}$ \\ ${ }^{4}$ Médico Cirurgião Geral
}

${ }^{1}$ Curso de Medicina, Universidade Brasil Campus Fernandópolis, 15600-000 Fernandópolis - SP, Brasil

${ }^{2}$ Curso de Medicina, Universidade Metropolitana de Santos - UNIMES 11015-470 Santos-SP, Brasil

${ }^{3}$ Residência em Cirurgia Geral, Santa Casa de Misericórdia de Fernandópolis, 15601-012 Fernandópolis - SP, Brasil

\section{Resumo}

A trombose venosa mesentérica é uma causa rara de isquémia mesentérica, potencialmente letal. A sua apresentação é insidiosa, o que dificulta o seu diagnóstico em fase inicial. Sexo masculino, recebido em unidade de urgência com dor em região periumbilical há 15 dias evoluindo para dor intensa há 4 dias. Procedeu-se ao exame do abdome que se apresentou globoso, flácido e doloroso a palpação em lado direito com ruídos hidroaéreos presentes. Já a tomografia de abdômen total, apresentou derrame pleural, borramento da gordura com formação de coleção em região de hipocôndrio direito, presença de trombo em veia porta, placas de calcificações em parede de aorta, artérias ilíacas e femorais, cisto cortical em rim esquerdo. Foi diagnosticado com trombose em veia mesentérica superior. A conduta adotada foi laparotomia exploradora, enterectomia parcial e enterro anastomose, em caráter de urgência. Realizado incisão xifo-púbica, identificada isquemia de alça de delgado $20 \mathrm{~cm}$ da válvula íleo cecal, feita enterectomia de $40 \mathrm{~cm}$, com saída de trombos de vasos ressecados, ligadura e verificada hemostasia, confeccionada anastomose entérica término terminal. Após vinte e nove dias, obteve alta hospitalar. Em termos gerais a trombose de veia mesentérica apresenta-se clinicamente sob as formas: aguda, subaguda ou crónica. Na forma aguda a sintomatologia é exuberante, desproporcional e com rápida evolução. Na subaguda a dor abdominal persiste ao longo de dias ou semanas. Na forma crónica não ocorrem sintomas durante a instalação de trombose. Destaca-se a importância dos exames de imagem para o diagnóstico

Descritores: Oclusão Vascular Mesentérica; Dor Abdominal; Serviços Médicos de Emergência.

\section{Abstract}

Mesenteric venous thrombosis is a rare cause of potentially lethal mesenteric ischemia. Its presentation is insidious, which makes its early diagnosis difficult. Male gender, received in an emergency unit with pain in the periumbilical region for 15 days and evolving to severe pain for 4 days. Abdominal examination was performed, which was globose, flaccid and painful, palpation on the right side with airborne noises. On the other hand, total abdomen tomography showed pleural effusion, fat blurring with collection formation in the right hypochondrium region, presence of portal vein thrombus, aortic wall calcification plaques, iliac and femoral arteries, cortical cyst in the left kidney. He was diagnosed with upper mesenteric vein thrombosis. The approach adopted was exploratory laparotomy, partial enterectomy and anastomosis burial, as a matter of urgency. A xipho-pubic incision was made, and a small $20 \mathrm{~cm}$ ischemia of the caecal ileum valve was identified. A 40 $\mathrm{cm}$ enterectomy was performed, with resected thrombi of the dried vessels, ligation and hemostasis, and an enteric anastomosis was made at the terminal end. After twenty-nine days, he was discharged from hospital. In general terms mesenteric vein thrombosis is clinically presented as: acute, subacute or chronic. In the acute form the symptoms are exuberant, disproportionate and rapidly evolving. In subacute abdominal pain persists over days or weeks. In chronic form no symptoms occur during the installation of thrombosis. The importance of imaging exams for the diagnosis is highlighted.

Descriptors: Mesenteric Vascular Occlusion; Abdominal Pain; Emergency Medical Services.

\section{Resumen}

La trombosis venosa mesentérica es una causa rara de isquemia mesentérica potencialmente letal. Su presentación es insidiosa, lo que dificulta su diagnóstico temprano. Sexo masculino, recibido en una unidad de emergencia con dolor en la región periumbilical durante 15 días y evolucionando a dolor intenso durante 4 días. Se realizó un examen abdominal, que era globoso, flácido y doloroso, palpación en el lado derecho con ruidos presentes en el aire. Por otro lado, la tomografía total de abdomen mostró derrame pleural, desenfoque de grasa con formación de acumulación en la región del hipocondrio derecho, presencia de trombo de la vena porta, placas de calcificación de la pared aórtica, arterias ilíacas y femorales, quiste cortical en el riñón izquierdo. Fue diagnosticado con trombosis de la vena mesentérica superior. El enfoque adoptado fue la laparotomía exploratoria, la enterectomía parcial y el entierro de anastomosis, como una cuestión de urgencia. Se realizó una incisión xifo-púbica, se identificó una pequeña isquemia de $20 \mathrm{~cm}$ de la válvula íleon cecal, se realizó una enterectomía de $40 \mathrm{~cm}$, con trombos resecados de los vasos secos, ligadura y hemostasia, y se realizó una anastomosis entérica en el extremo terminal. Después de veintinueve días, fue dado de alta del hospital. En términos generales, la trombosis de la vena mesentérica se presenta clínicamente como: aguda, subaguda o crónica. En la forma aguda, los síntomas son exuberantes, desproporcionados y evolucionan rápidamente. En el dolor abdominal subagudo persiste durante días o semanas. En forma crónica no se presentan síntomas durante la instalación de trombosis. Se destaca la importancia de los exámenes de imágenes para el diagnóstico.

Descriptores: Oclusión Vascular Mesentérica; Dolor Abdominal; Servicios Médicos de Urgencia.

\section{INTRODUÇÃO}

A trombose venosa mesentérica é uma entidade rara, que representa cerca de 10 a $15 \%$ das isquemias mesentéricas, afetando em $95 \%$ dos casos a veia mesentérica superior. Na maioria dos casos se identifica um ou mais fatores etiológicos, incluindo os estados de hipercoagulabilidade hereditários apresentando déficits de inibidores de coagulação ou aumento do nível ou função de fatores de coagulação. 
Em outros casos, podem ser adquiridos, em casos de neoplasias, uso de contraceptivos orais, síndrome nefrótica ou processos inflamatórios adjacentes, hipertensão portal, e após traumatismo ou cirurgia abdominal. Sua apresentação clínica é subtil e insidiosa, de difícil reconhecimento pela inespecifidade dos sintomas e sinais. $\mathrm{O}$ atraso no diagnóstico leva a morbilidade e mortalidade, as quais devem ser consideradas ${ }^{1}$.

Tal patologia foi reconhecida como causa de gangrena intestinal há mais de um século, envolvendo, preferencialmente, a veia mesentérica superior. Dessa maneira, pode ter um início agudo, subagudo ou crônico. A forma aguda se assemelha à isquemia arterial intestinal aguda, com dor abdominal súbita e desproporcional aos achados do exame físico. A crônica não costuma apresentar quaisquer sintomas até a ocorrência de complicações tardias. A forma subaguda se desenvolve ao longo de semanas a meses, porém sem determinar infarto intestinal desde o início. As manifestações clínicas dependem do tamanho e da extensão do trombo, bem como da profundidade da isquemia na parede intestinal ${ }^{2}$.

$\mathrm{Na}$ sua fisiopatologia tem-se o envolvimento com a tríade de Virchow: estase venosa (diminuição no fluxo sanguíneo), lesão do endotélio vascular e hipercoagulabilidade. Como consequência, leva à congestão da parede intestinal, edema, hemorragia intramural, necrose hemorrágica, causando manifestações clínicas de peritonite. Em razão do seu difícil diagnóstico, a trombose de veia superior pode levar a desfechos com alta mortalidade ${ }^{3}$.

Considerando a importância do tema, esse estudo visa relatar um caso de trombose mesentérica superior em paciente adulto e revisar o encontrado acerca desse tema na literatura.

\section{CASO CLÍNICO}

Paciente do sexo masculino, 58 anos, recebido em unidade de urgência com dor em região periumbilical há 15 dias evoluindo para dor intensa nos últimos 4 dias com irradiação para o dorso e piora após ingesta de alimentos e líquidos. Em resposta ao exame físico, observou-se regular estado geral, consciente, orientado, corado, acianótico, anictérico, afebril. Ao exame abdominal observou-se abdômen globoso, flácido, com dor à palpação em lado direito e ruídos hidroaéreos presentes.

A tomografia de abdômen total apresentou derrame pleural, borramento da gordura com formação de coleção em região de hipocôndrio direito, presença de trombo em veia porta, placas de calcificações (parede de aorta, artérias ilíacas e femorais) e cisto cortical em rim esquerdo. Foi diagnosticado trombose em veia mesentérica superior e a conduta adotada foi laparotomia exploradora, enterectomia parcial e enterro anastomose, em caráter de urgência.
Realizada incisão xifo-púbica, foi identificada isquemia de alça de delgado aproximadamente de $20 \mathrm{~cm}$ da válvula íleo cecal; feita enterectomia de $40 \mathrm{~cm}$, com saída de trombos de vasos ressecados, ligadura e verificada hemostasia, confeccionada anastomose entérica término terminal. Após a colocação de drenos, ocorreu o fechamento por planos. O paciente foi encaminhado para a unidade de terapia intensiva. No quarto dia foi transferido para a enfermaria onde iniciou com quadros de dor em flanco esquerdo tipo cólica, após a ingesta de líquidos e alimentos e episódios de diarreicos de forte odor e cor verde escuro.

Dessa maneira, retornou para a unidade de terapia intensiva após dez dias de pós-operatório apresentando fistula com baixo débito. Foram prescritos cuidados de terapia intensiva, medicamentos, terapia anticoagulante e dieta parenteral. Apresentou melhora do quadro e foi encaminhado para a enfermaria. Após vinte e nove dias, obteve alta hospitalar.

\section{DISCUSSÃO}

A maioria das causas de trombose mesentérica está associada a estados pró-trombóticos, consequentes de desordens de coagulação herdados ou adquiridos, câncer, condições inflamatórias intraabdominais, pós-operatório, cirrose e hipertensão portal. Sua manifestação clínica depende da extensão do trombo, tamanho dos vasos acometidos e da invasão das camadas da parede intestinal. A apresentação clínica clássica é a dor abdominal tipo cólica em mesogástrico, náusea, vômito, anorexia e diarreia, são mais comuns ${ }^{4}$.

A proteína $\mathrm{C}$ é um anticoagulante endógeno, dependente da vitamina $\mathrm{K}$, ativada após a ligação da trombina com trombomodulina, receptor endotelial, e inibe a coagulação, fazendo a clivagem dos fatores Va e VIIIa, potencializadas pela proteína $S$, atuando como um cofator não enzimático. Sob esse aspecto, pode-se prever as deficiências dos ainticoagulantes naturais, proteínas $\mathrm{C}$ e $\mathrm{S}$, que são associadas ao estado de hipercoagubilidade e a um risco aumentado de tromboembolismo venoso ${ }^{4}$.

De um modo geral a trombose da veia mesentérica apresenta-se clinicamente sob as formas aguda, subaguda ou crônica. Na forma aguda a sintomatologia inicial é exuberante, desproporcional aos achados físicos e com rápida evolução. $\mathrm{Na}$ subaguda a dor abdominal persiste ao longo de dias ou semanas. $\mathrm{Na}$ forma crônica não ocorrem sintomas durante a instalação de trombose, manifestando-se por sinais de hipertensão portal. Os achados laboratoriais são inespecíficos, sendo que a leucocitose e acidose aparecem em fase tardia. Destaca-se a importância dos exames de imagem para o diagnóstico. Há, ainda, associação com trombofilia, subjacente: déficit de proteína $\mathrm{C}$, déficit de 
antitrombina III e síndrome anti-fosfolipídico. A cirurgia está indicada para os casos em que existe evidência clínica ou imaginológica de perfuração ou isquemia, quando não há necrose o tratamento é a anticoagulação ${ }^{5}$.

Os sintomas de trombose de veia mesentérica superior são geralmente inespecíficos, nas quais incluem dor abdominal, náusea, vômito, diarreia e sangramento gastrointestinal ${ }^{5}$.

Realizado o diagnóstico, inicia-se a terapia com a anticoagulação com heparina, constituindo assim um pilar básico para o tratamento. A cirurgia fica reservada a casos sugestivos de infarto intestinal, sempre acompanhados de anticoagulação. Dessa maneira, deve ser levada em conta no diagnóstico diferencial de qualquer paciente com sintomas abdominais, especialmente se houver um fator de risco associado. A não especificidade dos sintomas, faz com que o diagnostico seja retardado. Um alto índice de suspeita, o diagnostico precoce com a ajuda de técnicas modernas de diagnóstico (ultra-som, tomografia computadorizada abdominal), precoce e tratamento com anticoagulação e cirurgia, se necessário, ajuda a melhorar a sobrevivência e evitar recorrências 6 .

No diagnóstico por meio de imagens é possível observar que os trombos localizam-se geralmente na origem ou no terço proximal do vaso, enquanto os êmbolos são causas de oclusão mais frequente na porção distal do vaso. Alguns fatores podem predispor a formação desses êmbolos e consequente oclusão mesentérica. Associam-se a fatores de risco, tais como: idade avançada, doença coronariana, fibrilação auricular, valvulopatia, disritmia, status pos infarto do miocárdio, historia de eventos trombo-embolicos, cirurgia aórtica, aortografia, angiografia coronariana, distúrbios de coagulação, dissecção aórtica ${ }^{7}$.

Pacientes com idade avançada e múltiplas comorbidades cardiovasculares podem aumentar o risco operatório, e a evolução do quadro clínico é frequentemente superior a 24 horas após a admissão no serviço de urgência, o que representa um fator de diagnostico desfavorável nesse grupo de pacientes. Tal patologia está associada à mortalidade mais elevada, pela maior extensão de intestino em sofrimento. Dessa maneira, todos são submetidos à laparotomia, com ressecção e a cirurgia de revascularização. $O$ conduto venoso deve ser preferido quando existe necrose intestinal ou mesmo na ausência quando se suspeita de contaminação abdominal ${ }^{8}$.

A revascularização mesentérica está indicada na presença de sintomas, e raramente é realizada como medida profilática, uma vez que o risco de isquemia intestinal na ausência dos sintomas é mínimo. O tratamento objetiva aliviar sintomas, restaurar o peso normal e prevenir o infarto intestinal $^{9}$. A realização de ultrassonografia abdominal, à entrada do paciente no serviço de urgência, permite identificar aspectos sugestivos de obstrução vascular. O diagnóstico de trombose será confirmado após realização de tomografia computadorizada, o que antecipa o diagnóstico, contribuindo para se obter uma melhor terapêutica do caso $^{7}$.

Logo, ressalta-se a importância da suspeição clinica e da necessidade de melhorar a referenciação, para que se possa encurtar a demora diagnóstica, como passos essenciais para melhores resultados terapêuticos. O sucesso depende de um forte índice de suspeição diagnostica associado à atitude cirúrgica de revascularização e ressecção intestinal ${ }^{8}$.

\section{REFERÊNCIAS}

1. Oliveira PH, Oliveira AH, Costa M, Silva A. Trombose Venosa Mesentérica: uma causa rara de oclusão intestinal. Rev Port Cir. 2012;22:61-6.

2. Felga GEG, Forn CG, Pinto JRF, Chebli JMF, Gaburri PD, Souza AFM et al. Trombose venosa mesentérica subaguda. HU Revista. 2003;29(3):488-89.

3. Bassani E, Iglesias GA, Souza MAR, Funes H, Funes F. Trombose Venosa Mesentérica em paciente com apendicite aguda. 2014. Disponível em: <https://faceres.com.br/wp-content/ uploads/ 2014/01/trombose-venosa-mesenterica-empacientes-com-apendicite-aguda.pdf $>$. Acesso em: 09 fev. 2019.

4. Simão JL, De Nadai LC, Giacon PP, Lopes MAM. Uso de contraceptivos orais induzindo trombose mesentérica. Rev Bras Hematol Hemoter. 2008;30(1):75-7.

5. Artifon ELA, Couto-Júnior DS, Fraga GP, Sakai O, Rasslan S. Endoscopic ultrasound (EUS) diagnosis of blunt pancreatic trauma associated to the superior mesenteric vein thrombosis. $\mathrm{ABCD}$, arq. bras. cir. dig. 2010;23(1):64-6.

6. Muñoz S, Cubo P, González-Castillo J, Nuevo JA, Garcia-Lamberechts EJ, Sanz A. Superior mesenteric venous thrombosis: a retrospective study of thirteen cases. Rev. esp. enferm. dig. 2004;96(6):385-94.

7. Caldeira A, Martin F, Pereira E, Sousa R, Gonçalves R, Tristan J et al . Contribuição de Ultrasonografia Abdominal no diagnóstico da Trombose Mesentérica. J Port Gastrenterol. 2009; 16(4):154-58.

8. Tiago J, Ministro A, Nunes JS, Fernandes JF. Isquémia Mesentérica Aguda: cinco anos de experiência institucional (2007-12). Angiol Cir Vasc. 2013;9(1):11-6.

9. Flis V, Mrdža B, Štirn B, Milotic F, Kobilica N, Bergauer A. Revascularização da artéria mesentérica superior isolada para tratamento de isquemia mesentérica crônica. Wiener Klinische Wochenschrift, 2015. 128(3-4):109-13. 
http://dx.doi.org/10.21270/archi.v8il2.4806

\section{CONFLITO DE INTERESSES}

Os autores declaram não haver conflitos de interesse.

\section{AUTOR PARA CORRESPONDENCIA}

Amanda Oliva Spaziani

spazianimedicina@gmail.com

Submetido em 29/09/2019

Aceito em 07/11/2019 